\title{
Aerosol stratification characterization of an astronomical site by means of a backscatter lidar at the Roque de los Muchachos Observatory
}

\author{
M. Sicard ${ }^{* a, b}$, S. Tomás ${ }^{a, b}$, M. N. Md Rebaa ${ }^{a, b}$, A. Comerón ${ }^{a}$, O. Batet ${ }^{a}$, C. Muñoz-Porcar ${ }^{a}$, A. \\ Rodriguez $^{\mathrm{a}}, \mathrm{F}$. Rocadenbosch ${ }^{\mathrm{a}, \mathrm{b}}$, C. Muñoz-Tuñón ${ }^{\mathrm{c}}$, J. J. Fuensalida ${ }^{\mathrm{c}}$ \\ ${ }^{a}$ Remote Sensing Laboratory/ Institut d'Estudis Espacials de Catalunya, Universitat Politècnica de \\ Catalunya, Barcelona, Spain; \\ ${ }^{\mathrm{b}}$ Institut d'Estudis Espacials de Catalunya - Centre de Recerca de l'Aeronàutica i de l'Espai / \\ Universitat Politècnica de Catalunya, Barcelona, Spain; \\ ${ }^{\mathrm{c}}$ Instituto de Astrofísica de Canarias, Tenerife, Spain
}

\begin{abstract}
The Roque de los Muchachos Observatory, located on the island of La Palma in the Canary Islands, is home of many astronomical facilities. In the context of the Extremely Large Telescope Design Study, an intensive lidar campaign was

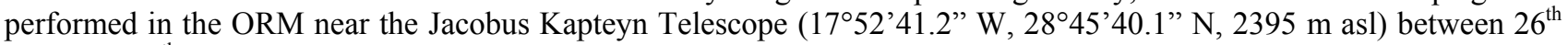
May and $14^{\text {th }}$ June 2008. The goal of the campaign was to characterize the atmosphere in terms of planetary boundary layer height and aerosol stratification vs. synoptic conditions. As a by-product an estimate of the aerosol optical thickness was also obtained and compared to the total atmospheric extinction coefficient measured by the Carlsberg Meridian Telescope.
\end{abstract}

Keywords: astronomical site characterization, lidar, aerosols, stratification, optical properties

\section{INTRODUCTION}

The Roque de los Muchachos Observatory (ORM), located in the municipality of Garafía on the northern part of the island of La Palma in the Canary Islands, is home of many astronomical facilities belonging to the European Northern Observatory. Most aerosols reaching the island of La Palma are either from marine/oceanic or African (Sahara and Sahel). From a previous study by Guerrero et al. [1] $25 \%$ of summer nights (June - September) are affected by African dust and only $10 \%$ the rest of the year. While it is well known that African dust can reduce significantly the visibility and therefore the quality of astronomical observations, the effect of marine/oceanic aerosols is not so well characterized and especially their vertical distribution and its effect on the quality of the observations is not known at all.

In the context of the Extremely Large Telescope (ELT) Design Study, an intensive lidar campaign was performed in the ORM near the Jacobus Kapteyn Telescope $\left(17^{\circ} 52^{\prime} 41.2^{\prime}\right.$ ' W, $28^{\circ} 45^{\prime} 40.1^{\prime \prime} \mathrm{N}, 2395 \mathrm{~m}$ asl) between $26^{\text {th }}$ May and $14^{\text {th }}$ June 2008 (hereinafter noted as $26 \mathrm{M}$ and $14 \mathrm{~J}$, respectively). The goal of the campaign was 1) to characterize the atmosphere in terms of planetary boundary layer (PBL) height and aerosol stratification vs. synoptic conditions, and 2) also to correlate the atmospheric structure with astronomical parameters tracers of the visibility and/or the turbulence. As a byproduct an estimate of the aerosol optical thickness (AOT) was also obtained and compared to the total atmospheric extinction coefficient measured by the Carlsberg Meridian Telescope (CMT) using the observations of about 30 to 40 calibrating stars per night.

The paper is organized as follows: Section 2 describes the instrumentation and the method used in the study; Sections 3 and 4 give the results of the aerosol stratification and loading in ORM, respectively, and finally Section 5 presents the conclusions of the study.

*msicard@tsc.upc.edu; phone 34-934011065; fax 34 934017200; www.tsc.upc.edu/rs/index.php

Remote Sensing of Clouds and the Atmosphere XIV, edited by Richard H. Picard, Klaus Schäfer,

Adolfo Comeron, Evgueni Kassianov, Christopher J. Mertens, Proc. of SPIE Vol. 7475, 74750P

(C) 2009 SPIE · CCC code: 0277-786X/09/\$18 - doi: 10.1117/12.833707

Proc. of SPIE Vol. 7475 74750P-1 


\section{INSTRUMENTATION AND METHOD}

The first day of the campaign, 26M, was dedicated to instrument set-up and testing. The measurements really started on the night of $27 \mathrm{M}$ and ended on the morning of $14 \mathrm{~J}$, i.e. over a period of 16 nights (during 2 nights, $30 \mathrm{M}$ and $31 \mathrm{M}$, the measurements had to be aborted because of low clouds at ground level).

The method employed to retrieve the aerosol stratification as well as the AOT by-product is based on lidar measurements performed during $27 \mathrm{M}-14 \mathrm{~J}$. Measurements from collaborative close-by instruments, as well as model (synoptic maps, backtrayectories, etc.) and satellite tools are also used. Table 1 shows the schedule of the measurements performed by the lidar. A total of 31 measurements of usually 60 -min. duration were performed. In some cases coinciding with relative close overpasses of the CALIOP (Cloud-Aerosol Lidar with Orthogonal Polarization) lidar on board of CALIPSO (Cloud-Aerosol Lidar and Infrared Pathfinder Satellite Observation) [2], the measurement was extended to $150 \mathrm{~min}$.

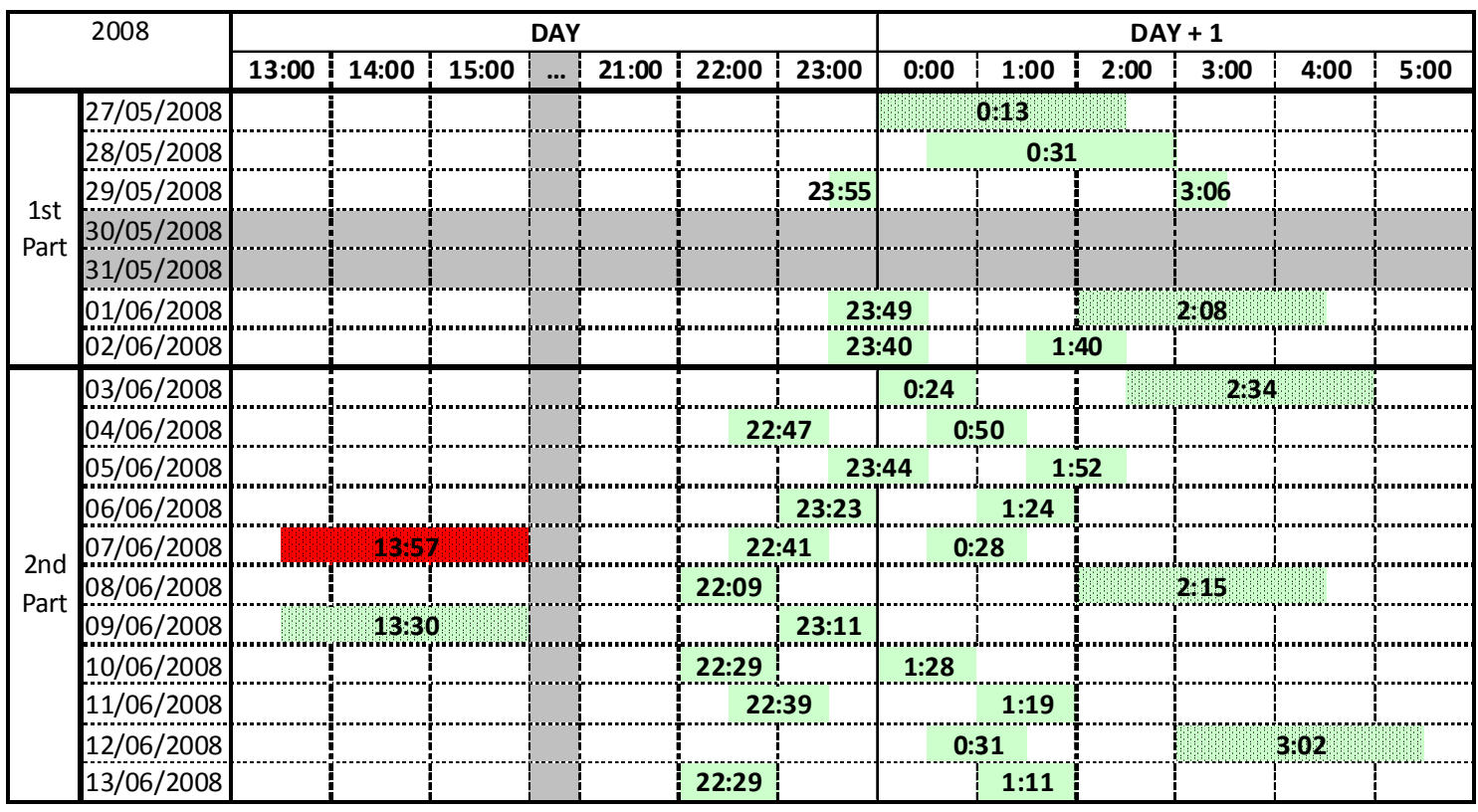

Table 1. Schedule of the measurements made at zenith during the campaign. The time is in UTC and indicates the starting time of the measurement. All regular measurements are 30- or 60-min. long; CALIPSO correlative measurements are 150-min. long and are indicated by dotted cells. The cell in red indicates profiles not usable because of acquisition problems.

\subsection{Lidar}

The lidar from the Universitat Politècnica de Catalunya (UPC) is based on a frequency-doubled Nd:YAG laser delivering simultaneously pulses of approximately $160 \mathrm{~mJ}$ and 7-ns duration at 1064 and $532 \mathrm{~nm}$ [3]. The backscattered light is collected by an 8-inch diameter Schmidt-Cassegrain telescope and focused on one end of an optical-fiber bundle. At the other end of the bundle dichroic beamsplitters deflect the collected light towards 3 photodetectors. An avalanche photodiode-based receiver is used for the 1064-nm channel. A photomultiplier tube-based receiver is used at both 532and $607.4 \mathrm{~nm}$ channels, the latter corresponding to the Raman shift of the incident radiation at $532 \mathrm{~nm}$ produced by the atmospheric nitrogen. The system full overlap factor is reached at approximately $400-500 \mathrm{~m}$.

The lidar was shipped from Barcelona to the Canary Islands by boat and installed near the Jacobus Kapteyn Telescope. Fig. 1a shows a detailed view of the UPC lidar optical head and Fig. 1b shows the system operating during nighttime in front of the Jacobus Kapteyn Telescope. Even though only one type of measurement is used in this work, two types of measurement were performed as follows:

- Nocturnal cycle: static measurement of usually 60-min. duration in a zenith line of sight (LOS) with a 1-min. resolution. The retrieved parameters are: AOT, vertical aerosol distribution, PBL height, and extinction and backscatter coefficient profiles. 
- Wind - horizontal: repeated (generally 60 times) alternative measurements between 2 LOS separated by an azimuth angle of 1 to $5^{\circ}$ (generally $5^{\circ}$ ). At each LOS the measurement is of 1.5- to 5-s duration (generally 3s). The elevation angle is 30 or $40^{\circ}$. The initial azimuth angle is $80^{\circ}$ compared to the North. The retrieved parameters are: PBL height (when the signal is not totally attenuated before it), horizontal components of wind velocity.

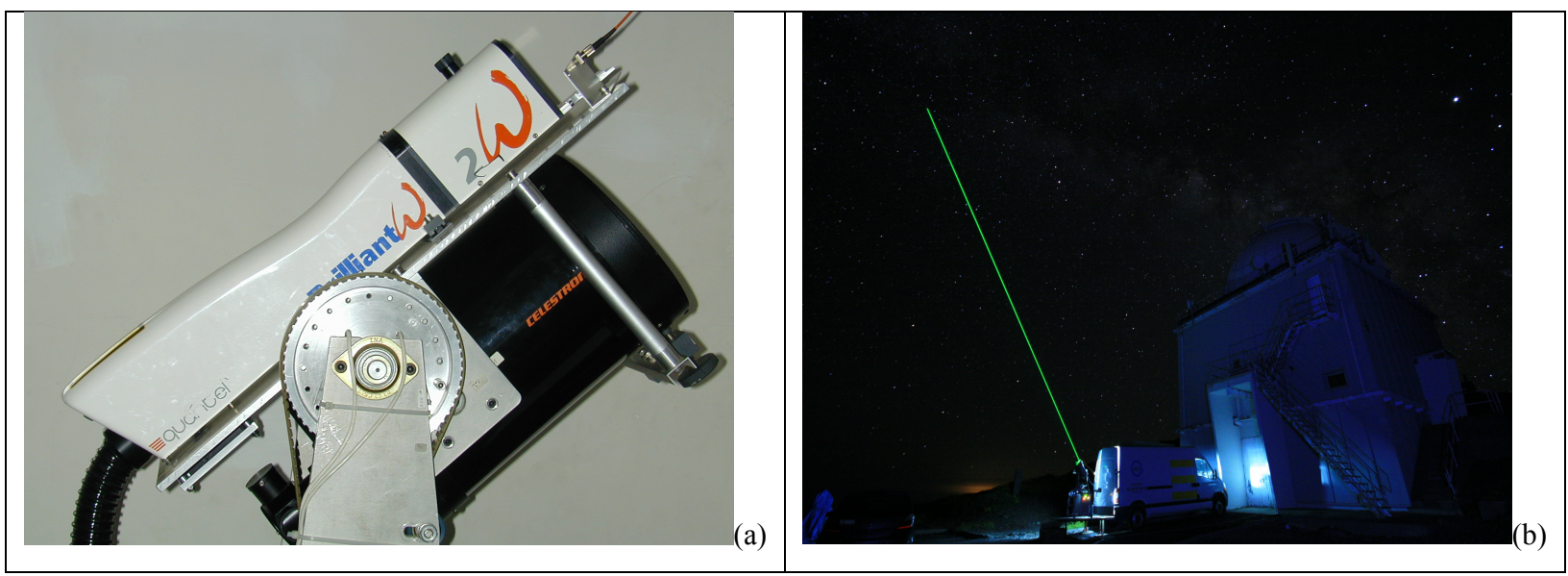

Fig. 1. (a) Detailed view of the UPC lidar optical head showing the laser on top of the telescope mounted on a motorized U-fork; (b) nighttime measurement in front of the Jacobus Kapteyn Telescope (courtesy of Pablo Bonet from the Instituto de Astrofísica de Canarias).

The retrieval of the PBL was made using the gradient method [4]. All profiles were visually inspected and the resulting PBL height was cross-compared to the previous and the following value in order to guarantee temporal coherency of its evolution. The retrieval of the aerosol optical coefficient profiles (backscatter and extinction) were retrieved by means of:

- The Klett-Fernald-Sasano method [5]-[7] using a constant lidar ratio of $30 \mathrm{sr}$.

- The Raman inversion method [8] for the 150-min. long measurements.

The AOT was measured by integrating the aerosol extinction coefficient profile from the minimum height of confidence, usually between 400 and $500 \mathrm{~m}$ agl, and up to the free troposphere, more specifically during the campaign up to heights varying between 3.1 and $6.8 \mathrm{~km}$ agl. The first part of the PBL not "seen" by the lidar, because of its incomplete overlap factor, was taken as the extrapolation of the extinction profile at the minimum height of confidence.

\subsection{Ground collaborative instruments}

\subsubsection{Total extinction coefficient from the Carlsberg Meridian Telescope}

From the observations of an average of $30-40$ photometric standard stars per night, the Carlsberg Meridian Telescope provides nightly values of the total extinction coefficient $\left(E C_{t o t}\right)$ in the Sloan Digital Sky Survey (SDSS) r' band at $\lambda_{r^{\prime}}=625 \mathrm{~nm}$ derived from CCD frames at the ORM. Since the type of aerosol observed above ORM is supposedly not known, the conversion of $E C_{t o t}$ at $\lambda_{r^{\prime}}$ into AOT at $\lambda_{\text {lidar }}$ is not straight forward. For that reason the comparison between both the lidar and the CMT measurements is made in terms of TOT , the total optical thickness. The calculation of $E C_{\text {tot }}$ at $\lambda_{\text {lidar }}=532 \mathrm{~nm}$ is made as follows. First the aerosol extinction coefficient $\left(E C_{a e r}\right)$ is calculated using the formula [9]:

$$
E C_{a e r}=E C_{t o t}\left(\lambda_{r^{\prime}}\right)-E C_{m o l}\left(\lambda_{r^{\prime}}\right),
$$

where $E C_{m o l}\left(\lambda_{r^{\prime}}\right)=0.0734$ mag airmass $^{-1}$ represents the molecular extinction coefficient at $\lambda_{r^{\prime}} \quad E C_{m o l}\left(\lambda_{r^{\prime}}\right)$ can be extracted from a look-up table given in [9] for any other wavelength. According to [10] in the range [300; $1100 \mathrm{~nm}$ ] 
$E C_{a e r}$ in ORM can be considered, to a first approximation, independent of the wavelength and therefore $E C_{\text {tot }}$ can then be expressed at any wavelength as:

$$
E C_{t o t}(\lambda)=E C_{a e r}+E C_{m o l}(\lambda)=E C_{t o t}\left(\lambda_{r^{\prime}}\right)-E C_{m o l}\left(\lambda_{r^{\prime}}\right)+E C_{m o l}(\lambda) .
$$

At $\lambda_{\text {lidar }}=532 \mathrm{~nm}, E C_{\text {mol }}\left(\lambda_{\text {lidar }}\right) \square E C_{\text {mol }}(530 \mathrm{~nm})=0.1087$ mag $^{\text {airmass }}{ }^{-1}$. The conversion from $E C_{\text {tot }}$ into TOT at $\lambda_{\text {lidar }}$ is then realized by simply dividing $E C_{\text {tot }}$ by the conversion factor 1.086 mag.airmass ${ }^{-1}$.

\subsubsection{In situ meteorological information}

Ground pressure, temperature, and humidity, as well as wind speed and direction were also provided by the weather station of the Isaac Newton Group of Telescopes situated a few hundreds meters away from the lidar.

\subsection{Satellite and model tools}

The DREAM (Dust Regional Atmospheric Model) model was used as well as MODIS (Moderate Resolution Imaging Spectroradiometer) images to discard the presence of Saharan dust during the nights of measurements.

Mean synoptic maps of sea level pressure were generated from the NCEP-NCAR (National Centers for Environmental Prediction-National Center for Atmospheric Research) reanalysis project [11].

\section{AEROSOL STRATIFICATION IN ORM}

\subsection{Synoptic situation}

According to a thorough study of the day-after-day evolution of synoptic maps of the sea level pressure provided by the NCEP-NCAR reanalysis project [11], it is possible to distinguish two main synoptic patterns during the duration of the campaign: $27 \mathrm{M}-02 \mathrm{~J}$ and $03 \mathrm{~J}-14 \mathrm{~J}$. Fig. 2 shows the average map at $0000 \mathrm{UTC}$ for both periods. The first half of the campaign was dominated by a stable anticyclone situation centered on the Azores islands or slightly southwest of them, a situation that can possibly be considered typical. In the second half of the campaign a new high pressure system coming from the Caribbean pushed the Azores anticyclone northeast towards Western Europe. These two situations bring air masses from different origin and identified according to [12] as Atlantic (marine/oceanic) during 27M - 02J and European (anthropogenic emissions from sulfates and carbon) during 03J - 14J.

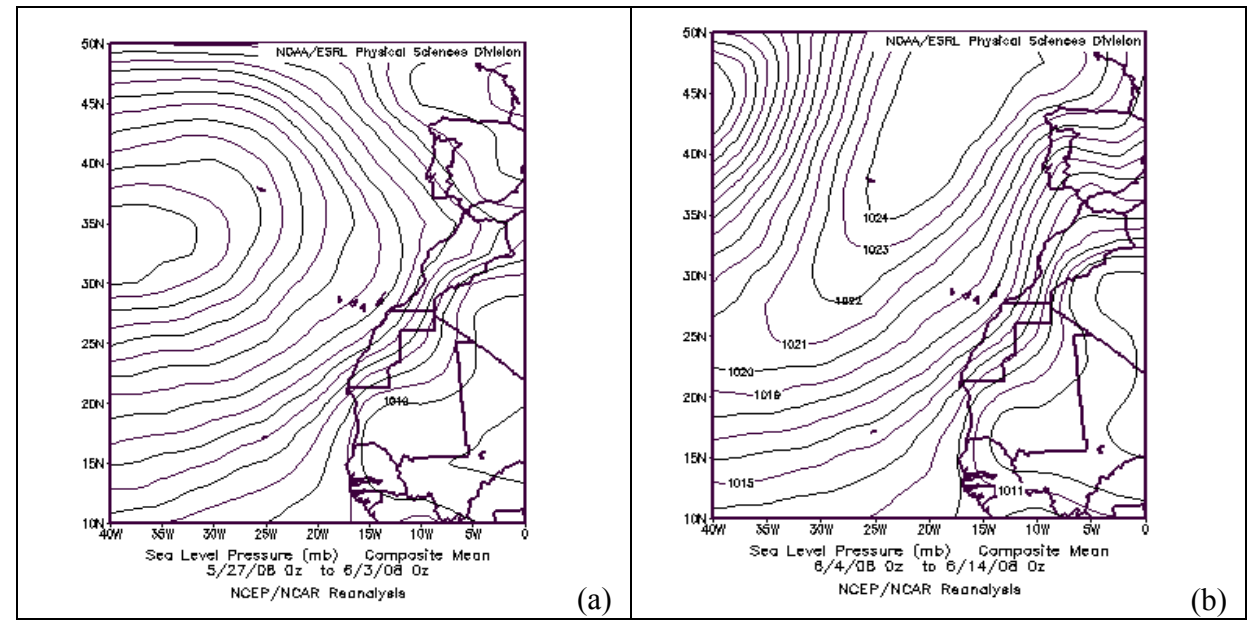

Fig. 2. Sea level pressure at 0000UTC averaged over the period (a) $27 \mathrm{M}-02 \mathrm{~J}$, and (b) $03 \mathrm{~J}-14 \mathrm{~J}$. 


\subsection{Aerosol stratification in ORM}

The temporal evolution of the range-square corrected signal (RSCS) at $1064 \mathrm{~nm}$ during the 16 nights of the campaign is shown in Fig. 3 and Fig. 4. The choice for representing the 1064-nm channel profiles comes from the higher contrast between aerosols and molecules that offers that wavelength compared to $532 \mathrm{~nm}$. Another issue to take into account for interpreting correctly those two figures is that the color bar scale is adjusted for every night in order to emphasize the signal variations in the PBL. Consequently according to the state of the PBL the molecular region (aerosol-free) appears or blue or yellow-green. The mean PBL height calculated every $30 \mathrm{~min}$. is also indicated by '+' signs as well as its error bar computed as the standard deviation of the 30 profiles used to calculate the mean PBL height. Mid- and high-altitude clouds (above $4.2 \mathrm{~km}$ agl) were present on two nights: $28 \mathrm{M}-29 \mathrm{M}$ and $29 \mathrm{M}-30 \mathrm{M}$. The main feature is that all figures show a multi-layer stratification: during the first part of the campaign a single layer is detected above the PBL while in the second part several layers are observed. During the whole campaign the nocturnal cycles show in general a low nocturnal PBL at a mean height of $543 \pm 39 \mathrm{~m}$ agl and always lower than $1000 \mathrm{~m}$ agl. During the first and the second part of the campaign the mean PBL height is around $487 \pm 30 \mathrm{~m}$ and $571 \pm 82 \mathrm{~m}$ agl, respectively. These heights are slightly higher than measurements made in June - July 2007 by Sicard et al. [13] in the Teide Observatory on the neighboring island of Tenerife at $2400 \mathrm{~m}$ asl where the PBL heights fluctuated between 375 and $810 \mathrm{~m}$ agl.

Fig. 5 shows the temporal evolution of the PBL height during the whole campaign, as well as the highest aerosol layer detected by the lidar. The average of the latter layer is around $3349 \mathrm{~m}$ agl. During the first and the second part of the campaign it rises up to 1983 and $4008 \mathrm{~m}$ agl, respectively, which corresponds to 4378 and $6403 \mathrm{~m}$ asl. In terms of aerosol distribution above sea level these layers are remarkably high.

\section{AEROSOL LOADING AND DISCUSSION}

The lidar AOT was retrieved at both wavelengths of 1064 and $532 \mathrm{~nm}$. However in approximately half of the cases the inversion at $532 \mathrm{~nm}$ failed due to too weak signals of the aerosol return of the order of magnitude of the error range. Fig. 6 shows the temporal evolution of the AOT, which is very low at both lidar wavelengths: the average values are 0.04 and 0.0045 at 532 and $1064 \mathrm{~nm}$, respectively. The average AOTs for the first and for the second part of the campaign are, respectively, 0.05 and 0.03 at $532 \mathrm{~nm}$, and 0.008 and 0.003 at $1064 \mathrm{~nm}$. In the second part of the campaign, the AOT at $1064 \mathrm{~nm}$ seems to be rather constant over the whole period. In spite of more extended aerosol vertical distributions in the second part of the campaign the AOT results lower than in the first part indicating that the highest layers do not contribute significantly to the optical thickness in the atmospheric column. Since the PBL height did not vary significantly between both periods, it also indicates that the type of aerosols contained in the PBL, or at least their optical properties, was different for both periods. A striking difference between both periods was observed on the continuous measurements of humidity at ground level performed at the meteorological station of the Isaac Newton Group of Telescopes: the average value was approximately between $50-75 \%$ and below $25 \%$ during the first and the second part of the campaign, respectively. The aerosol higroscopicity might explain the reason why higher AOTs were found in the first part of the campaign compared to the second period. As an example, Shettle and Fenn [14] reported an increase of the extinction coefficient of marine aerosols of 20 and $40 \%$ at 532 and $1064 \mathrm{~nm}$, respectively, when the relative humidity was increased from 10 to $70 \%$.

The comparison between the lidar-derived and the CMT-derived TOT at $532 \mathrm{~nm}$ over the whole campaign is shown in Fig. 7. The minimum value of the CMT-derived TOT is 0.1089 and apart from this value all the others are higher than 0.1105 which represents the upper limit indicating no atmospheric extinction at all. Therefore the CMT confirms the presence of aerosols on all nights. Additionally it also confirms the absence of Saharan dust since the TOT does not exceed (except in one case on 28M at 0031 UTC) the lower limit indicating the presence of Saharan dust fixed to 0.1409 by Guerrero et al. [1] (and converted in TOT in the present paper). The humidity at ground level revealed that on the night of $27 \mathrm{M}-28 \mathrm{M}$ exceeded $50 \%$ and that before $2200 \mathrm{UTC}$ it even reached $100 \%$. The mean value of CMT-derived TOT over the whole campaign is 0.1172 which is a relatively large value compared to the mean value of 0.104 calculated over 15 years (1984-1998) by Guerrero et al. [1] (and converted in TOT here). This results also confirms the conclusion from the same authors that in summer high-extinction episodes are more frequent than the rest of the year. The mean CMT-derived TOT over the first part of the campaign, 0.123 , is slightly larger than over the second part, 0.115. From Fig. 7, the lidar-derived TOT fluctuated between 0.123 and 0.186 while the CMT-derived TOT remains more or less constant around the average value of 0.1172. No correlation is found between both quantities probably because of the mixing state of the aerosols as also suggested by Varela et al. [12] when comparing $E C_{t o t}$ with 


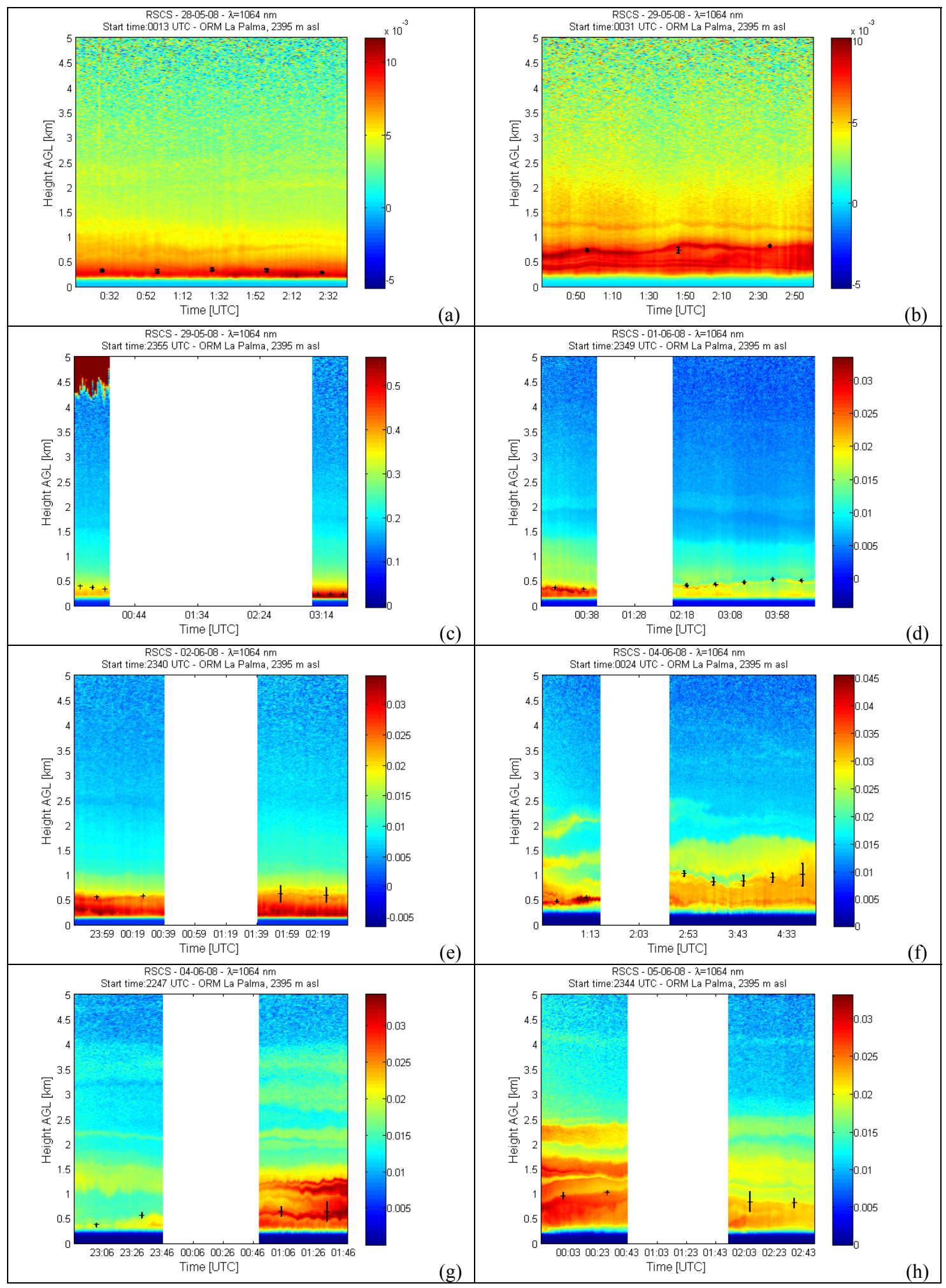

Fig. 3. RSCS at $1064 \mathrm{~nm}$ showing the aerosol stratification at ORM on the night of (a) $27 \mathrm{M}$, (b) $28 \mathrm{M}$, (c) $29 \mathrm{M}$, (d) $01 \mathrm{~J}$, (e) $02 \mathrm{~J}$, (f) $03 \mathrm{~J}$, (g) $04 \mathrm{~J}$ and (h) $05 \mathrm{~J}$. 


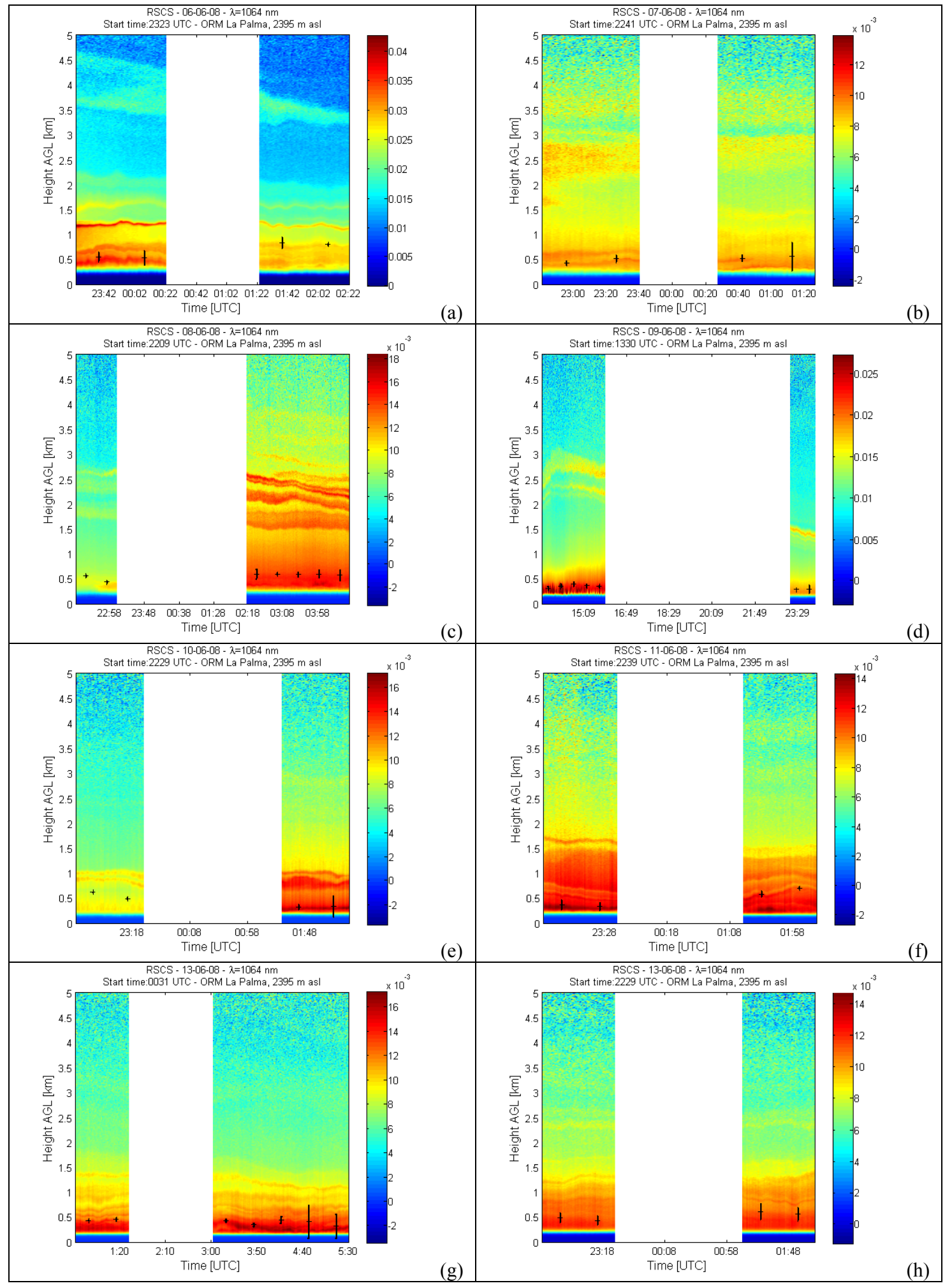

Fig. 4. RSCS at $1064 \mathrm{~nm}$ showing the aerosol stratification at ORM on the night of (a) 06J , (b) 07J, (c) 08J, (d) 09J, (e) 10J, (f) 11J, (g) $12 \mathrm{~J}$ and (h) $13 \mathrm{~J}$. 
the aerosol index provided by the spaceborne OMI (Ozone Monitoring Instrument) instrument. Another probable reason is the inconsistency of the hypothesis made that the $E C_{a e r}$ in ORM is independent of the wavelength.

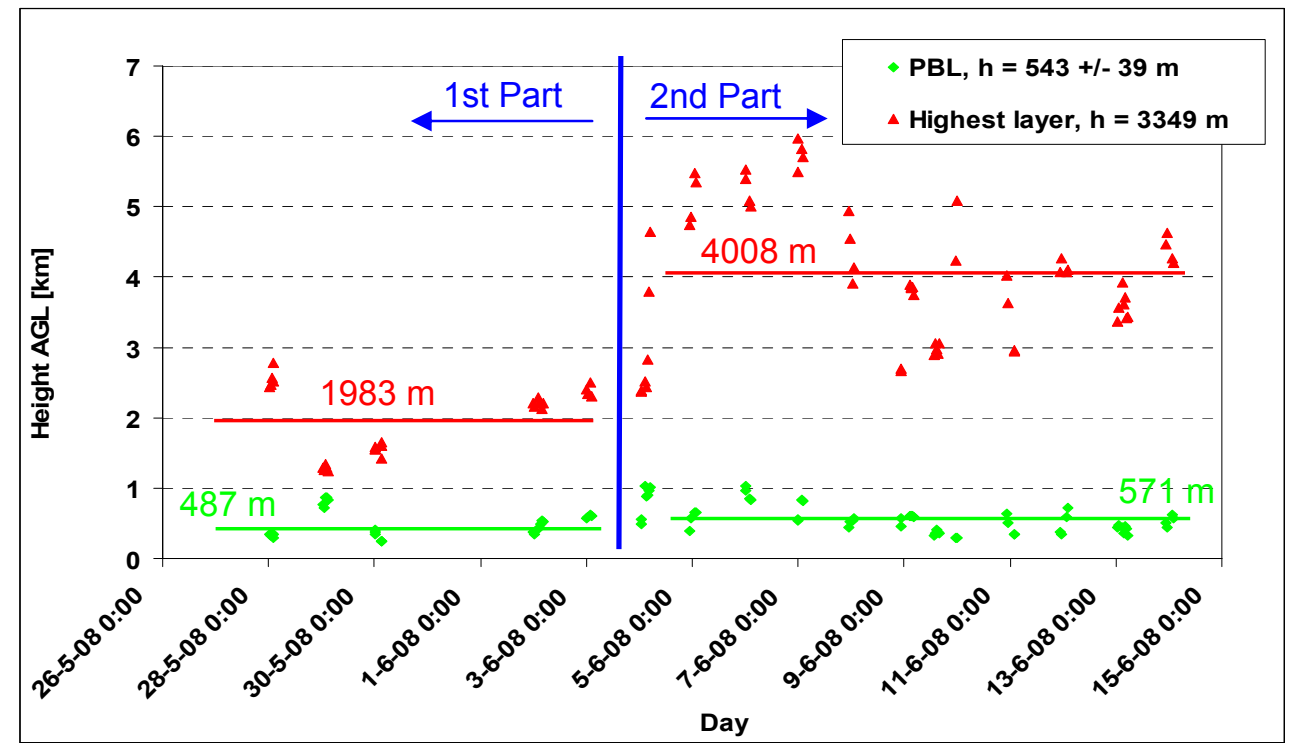

Fig. 5. Temporal evolution of the PBL height and of the highest aerosol layer detected. In the legend ' $h$ ' indicates the mean height over the whole campaign. The numbers in green and in red indicate, respectively, the mean PBL height and the mean height of the highest aerosol layer during $27 \mathrm{M}-02 \mathrm{~J}$ and $03 \mathrm{~J}-14 \mathrm{~J}$.

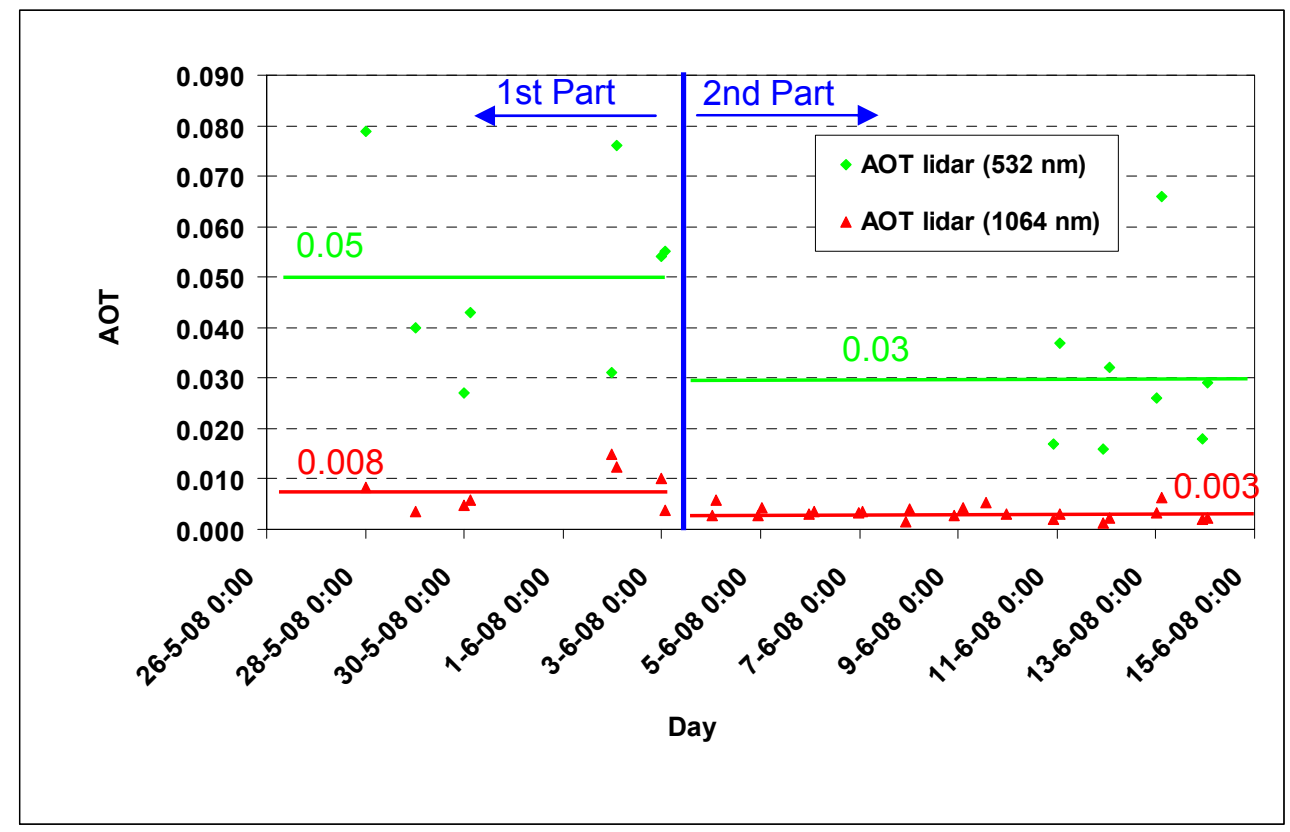

Fig. 6. Temporal evolution of the lidar-derived AOT at 532 and $1064 \mathrm{~nm}$. The numbers in green and in red indicate the mean AOT, respectively, at 532 and $1064 \mathrm{~nm}$ during $27 \mathrm{M}-02 \mathrm{~J}$ and $03 \mathrm{~J}-14 \mathrm{~J}$. 


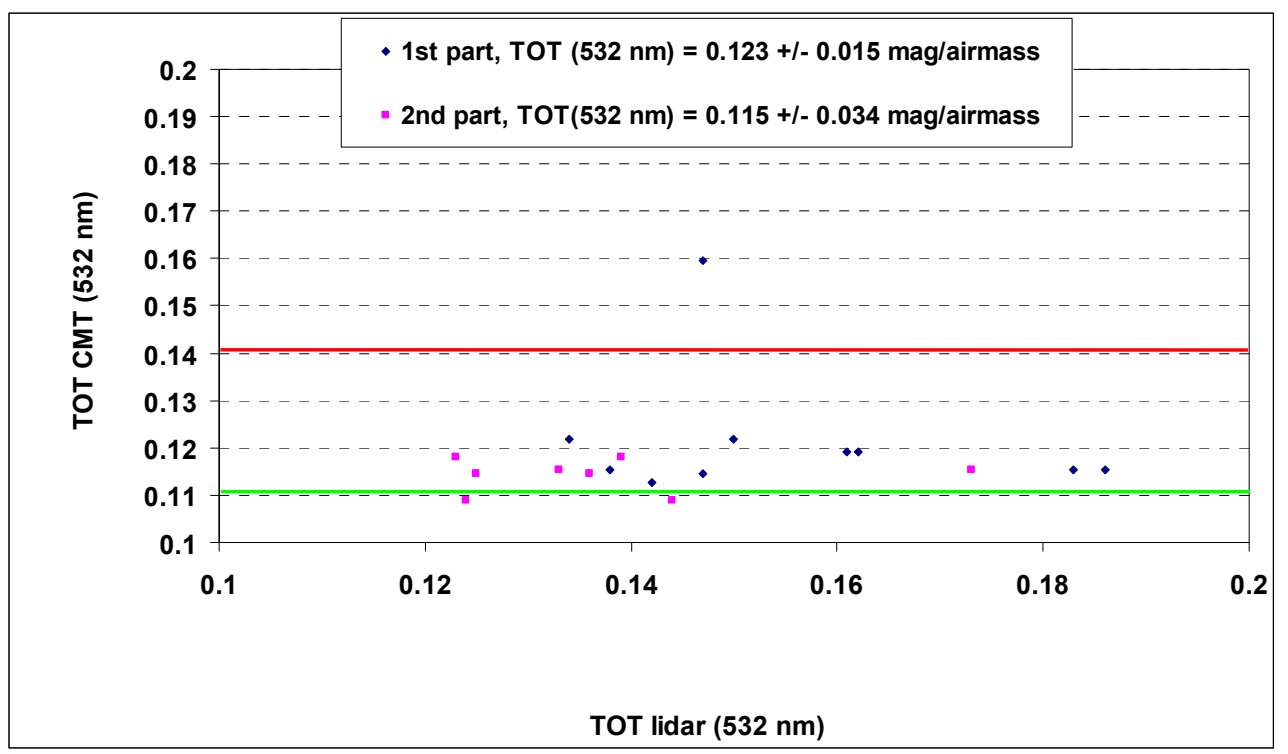

Fig. 7. CMT-derived vs. lidar-derived TOT at $532 \mathrm{~nm}$. Green and red lines indicate the TOT (532 nm) limits for no atmospheric extinction at all (0.1105) and Saharan dust presence (0.1409), respectively.

\section{CONCLUSION}

An astronomical site has been characterized in terms of aerosol stratification and loading by means of an aerosol, backscatter lidar in the framework of the "Extremely Large Telescope Design Study" European project. To our knowledge it is the first time that results from such an experiment are presented. A total of 31 measurements of at least 60-min. duration were performed during 16 nights at the Roque de los Muchachos Observatory at $2395 \mathrm{~m}$ asl during 26 May 2008 and 14 June 2008. Two main synoptic scenarios were identified bringing, supposedly, marine/oceanic aerosols in the first half of the campaign and European aerosols in the second half. In both scenarios the lidar nocturnal cycles revealed a multi-layer stratification. During the whole campaign the nocturnal PBL is relatively low at a mean height of $543 \pm 39 \mathrm{~m}$ agl and does not change significantly between the first and the second part of the campaign, whereas, respectively, the highest layer of aerosols has its top at 1983 and $4008 \mathrm{~m}$ agl, which corresponds to 4378 and $6403 \mathrm{~m}$ asl, which denotes remarkably elevated layers. As far as aerosol loading is concerned, the AOT is very low: the average values are 0.04 and 0.0045 at 532 and $1064 \mathrm{~nm}$, respectively. During the first part of the campaign the AOT was approximately two to almost three times higher than in the second part whereas the highest aerosol layers were detected at lower altitude. This indicates whether a higher aerosol concentration in the first part of the campaign or a quite different (more absorbing) aerosol composition. Another possible explanation might be the change in the aerosol humidity which at ground level was two to three times higher in the first part of the campaign compared to the rest of it. The average total extinction coefficient measured by the CMT instrument is $0.1273 \mathrm{mag}$-airmass ${ }^{-1}$. It is a relatively large value compared to previous statistical studies [1] which confirms the fact that in summer high-extinction episodes are more frequent than the rest of the year.

\section{ACKNOWLEDGMENTS}

This work is supported by the European Union under the projects "Technology development program towards a European Extremely Large Telescope" (ELT Design Study, EU Specific Support Action, contract n ${ }^{\circ} 011863$ (RIDS)), and EARLINET-ASOS (EU Coordination Action, contract $\mathrm{n}^{\circ} 025991$ (RICA)); by the MICINN (Spanish Ministry of Science and Innovation) and FEDER funds under the projects CIT-020400-2005-56 and CIT-020400-2007-67, the 
project TEC2009-09106/TEC and TEC2006-07850/TCM, and the Complementary Actions CGL2008-01330-E/CLI and CGL2007-28871-E/CLI; and by the European Space Agency under the project 21487/08/NL/HE.

\section{REFERENCES}

[1] Guerrero, M.A., García-López, R.J., Corradi, R.L.M., Jiménez, A., Fuensalida, J.J., Rodríguez-Espinosa, J.M., Alonso, A., Centurión, M., Prada, F., "Extinction over the Canarian Observatories:the limited influence of Saharan dust", New Astronomy Reviews 42, 529 - 532 (1998).

[2] Winker, D., Pelon J., McCormick, P., "Initial Results from CALIPSO”, in Reviewed and Revised Papers Presented at the 23 ${ }^{\text {rd }}$ International Laser Radar Conference, C. Nagasawa and N. Sugimoto, Eds., Tokyo Metropolitan Univ., Tokyo, 991 - 994 (2006).

[3] Rocadenbosch, F., Sicard, M., Comerón, A., Baldasano, J.M., Rodríguez, A., Agishev, R., Muñoz, C., López, M.A., GarcíaVizcaino, D. , "The UPC scanning Raman lidar: an engineering overview", Proc. of the $21^{\text {st }}$ ILRC, Vol. 1, 69 - 70 (2002).

[4] Sicard, M., Pérez, C., Rocadenbosch, F., Baldasano, J.M. , García-Vizcaino, D., "Mixed-layer depth determination in the Barcelona coastal area from regular lidar measurements: methods, results and limitations", Boundary-Layer Metorol. 119,135 157 (2006).

[5] Klett, J. D., "Lidar inversion with variable backscatter/extinction ratios," Appl. Opt. 24, 1638 - 1643 (1985).

[6] Fernald, F. G., "Analysis of atmospheric lidar observations: some comments," Appl. Opt. 23, 652 - 653 (1984).

[7] Sasano, Y., Nakane, H., "Significance of the extinction/backscatter ratio and the boundary value term in the solution for the twocomponent lidar equation," Appl. Opt. 23, 11 - 13 (1984).

[8] Ansmann, A., Wandinger, U., Riebesell, M., Weitcamp, C., Michaelis, W., "Independent measurement of extinction and backscatter profiles in cirrus clouds by using a combined Raman elastic-backscatter lider", Appl. Opt. 31, 7113 - 7131 (1992).

[9] King, D. L., RGO/La Palma Technical Note 31 (1985).

[10] Jones, D. H. P., RGO/La Palma Technical Note 10 (1984).

[11] Kalnay, E., Kanamitsu, M., Kistler, R., Collins, W., Deaven, D., Gandin, L., Iredell, M., Saha, S., White, G., Woollen, J., Zhu, Y., Chelliah, M., Ebisuzaki, W. Higgins, W., Janowiak, J., Mo, K. C., Ropelewski, C., Wang, J., Leetmaa, A., Reynolds, R., Jenne, R., Joseph, D., “The NCEP/NCAR Reanalysis 40-year Project.”, Bull. Amer. Meteor. Soc. 77, 437 - 471 (1996).

[12] Varela, A.M., Bertolin, C., Muñoz-Tuñón, C., Ortolani, S., Fuensalida, J.J., “Astronomical site selection: On the use of satellite data for aerosol content monitoring”, Mon. Not. R. Astron. Soc. 391, 507 - 520 (2008).

[13] Sicard, M., Tomás, S., Comerón, A., Rocadenbosch, F., Rodríguez, A. Muñoz, C. Batet, O., "Planetary boundary layer height and wind field characterization by means of a lidar at the Teide observatory in the Canary islands", Proc. Of the IEEE International Geoscience and Remote Sensing Symposium (IGARSS-08), ISBN: 1-4244-2808-3, III-915 - III-918 (2008).

[14] Shettle, E. P., Fenn, R. W., "Models for aerosols of the lower atmosphere and the effects of humidity variations on their optical properties", Envisonmental Research paper No. 675, AFGL-TR-79-0214, NTIS, ADA 085951 (1979). 